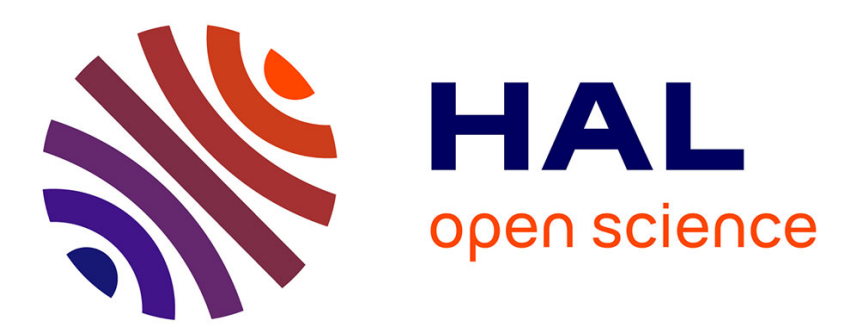

\title{
On Computing Algebraic Functions using Logarithms and Exponentials
}

\author{
Dima Grigoriev, Michael Singer, Andrew Yao
}

\section{To cite this version:}

Dima Grigoriev, Michael Singer, Andrew Yao. On Computing Algebraic Functions using Logarithms and Exponentials. SIAM Journal on Computing, 1995. hal-03049482

\section{HAL Id: hal-03049482 \\ https://hal.science/hal-03049482}

Submitted on 9 Dec 2020

HAL is a multi-disciplinary open access archive for the deposit and dissemination of scientific research documents, whether they are published or not. The documents may come from teaching and research institutions in France or abroad, or from public or private research centers.
L'archive ouverte pluridisciplinaire HAL, est destinée au dépôt et à la diffusion de documents scientifiques de niveau recherche, publiés ou non, émanant des établissements d'enseignement et de recherche français ou étrangers, des laboratoires publics ou privés. 


\title{
On Computing Algebraic Functions using Logarithms and Exponentials
}

\author{
Dima Grigoriev ${ }^{1}$, Michael Singer ${ }^{2}$, and Andrew $\mathrm{Yao}^{3}$
}

\begin{abstract}
Let $\rho$ be a set of algebraic expressions constructed with radicals and arithmetic operations, and which generate the splitting field $F$ of some polynomial. Let $N_{\beta}(\rho)$ be the minimum total number of root-takings and exponentiations used in any straightline program for computing the functions in $\rho$ by taking roots, exponentials, logarithms, and performing arithmetic operations. In this paper it is proved that $N_{\beta}(\rho)=\nu(G)$, where $\nu(G)$ is the minimum length of any cyclic Jordan-Hölder tower for the Galois group $G$ of $F$. This generalizes a result of Ja'Ja' [1], and shows that the inclusion of certain new primitives, such as taking exponentials and logarithms, does not improve the cost of computing such expressions as compared with programs which use only root-takings.
\end{abstract}

\footnotetext{
${ }^{1}$ Departments of Computer Science and Mathematics, Pennsylvania State University, University Park, Pennsylvania 16802.

${ }^{2}$ Department of Mathematics, North Carolina State University, Raleigh, North Carolina 27695-8205. Partially supported by NSF Grant DMS 90-24624.

${ }^{3}$ Department of Computer Science, Princeton University, Princeton, New Jersey 08544.
} 


\section{Introduction}

The question of how efficiently one can evaluate expressions such as $\left(\sum_{1 \leq i<j \leq n} \sqrt{\left(x_{i}-x_{j}\right)^{2}+\left(y_{i}-y_{j}\right)^{2}}\right) /\left(\begin{array}{c}n \\ 2\end{array}\right)$, the mean distance among $n$ points in the plane, was raised in Shamos and Yuval [8]. A systematic study of this question was given in Pippenger [6,7]. Let $\rho$ be a family of algebraic expressions constructed from indeterminates using radicals and arithmetic operations. Define the cost of a program to be the number of root-takings used, with arithmetic operations given for free. Let $\mathbf{F}$ be the extension field generated by the members of $\rho$ over the field of rational functions with complex coefficients. It was shown $[6,7]$ that, when the members of $\rho$ are rational functions of the roots of rational functions, the mimimum cost is equal to the number of the torsion orders $^{4}$ for the Galois group of $\mathbf{F}$ (an Abelian group in this case). An extension was given in Ja'Ja' [1], who showed that the minimum cost is equal to the minimum length of any cyclic Jordan-Hölder tower for the Galois group of $\mathbf{F}$, provided that $\mathbf{F}$ is a finite Galois extension over the field of rational functions. It is known $[1,7]$ that the former result is a special case of the latter.

These results can be used to determine the minimum cost for computing $\rho$ in many cases. For example, for the mean distance problem, the Galois group can be shown [6] to

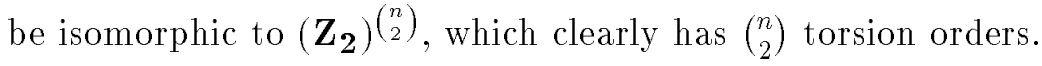

As taking a root $y^{1 / d}$ can be simulated by taking the logarithm $\log y$ followed by an exponentiation $\exp ((\log y) / d)$, a natural question is whether the availability of the lograrithm and exponential operations can substantially reduce the cost of evaluating algebraic expressions. In particular, can one evaluate the expression $\sum_{1 \leq i \leq n} \sqrt{x_{i}}$ using $o(n)$ exponentiations and logarithm-takings? (Clearly, the expression can be evaluated with $n$ root-takings.) The possible use of logarithms and exponentials, as well as other primitives, was mentioned in [8], but was not studied in later papers $[1,6,7]$.

In this paper, we show that under the same assumption as in [1] (i.e. $\mathbf{F}$ being a finite Galois extension), the availability of taking logarithms and exponentials does not reduce the cost. In particular, we prove that $n$ or more operations are needed to evaluate $\sum_{1 \leq i \leq n} \sqrt{x_{i}}$, with arithmetic operations given for free. In the next section, we give a precise statement of the main result (Theorem 1), after introducing the needed notations and background. The result is then proved in Section 3; some additional concepts and results from Differential Algebra (see [2-4]) are used in the proof.

\footnotetext{
${ }^{4}$ Any finite Abelian group $G$ can be uniquely decomposed into a direct sum of cyclic groups $\mathbf{Z}_{\mathbf{d}_{1}} \oplus$ $\mathbf{Z}_{\mathbf{d}_{\mathbf{2}}} \oplus \cdots \mathbf{Z}_{\mathbf{d}_{\mathbf{t}}}$, such that $d_{t}>1$ and $d_{i}$ is divisible by $d_{i+1}$ for $1 \leq i<t$. The integers $d_{i}$ are called the torsion orders of $G ; t$ is the number of torsion orders for $G$.
} 
We remark that the complexity question under other cost measures, in which the cost of taking a $d$-th root may depend on $d$, were discussed in $[1,6,7]$. We will not pursue it here.

\section{The Main Result}

We use the standard teminology in Algebra (as in Lang [3]). In what follows, let $\mathbf{Z}^{+}$be the set of all positive integers.

An $\alpha$-program $A$ is a sequence of instructions of the form $z_{1} \leftarrow I_{1}, z_{2} \leftarrow I_{2}, \cdots, z_{m} \leftarrow$ $I_{m}$, where $I_{i}$ are of the form $\left(r_{i}\left(x_{1}, x_{2}, \cdots, x_{n}, z_{1}, \cdots, z_{i-1}\right)\right)^{1 / d_{i}}$ with $r_{i}$ is a rational function in $x_{1}, \cdots, x_{n}, z_{1}, \cdots, z_{i-1}$ with complex coefficients and $d_{i} \in \mathbf{Z}^{+}$. We call $m$ the cost of $A$. For $1 \leq i \leq m$, let $g_{i}\left(x_{1}, x_{2}, \cdots, x_{n}\right)$ be the functions defined inductively by $g_{i}\left(x_{1}, x_{2}, \cdots, x_{n}\right)=\left(r_{i}\left(x_{1}, x_{2}, \cdots, x_{n}, g_{1}\left(x_{1}, \cdots, x_{n}\right), \cdots, g_{i-1}\left(x_{1}, \cdots, x_{n}\right)\right)\right)^{1 / d_{i}}$. We shall always assume that the $r_{i}$ have been chosen so that the denominators of these functions do not vanish identically. Informally, $g_{i}\left(x_{1}, x_{2}, \cdots, x_{n}\right)$ are the values assumed by the variables $z_{i}$ for input $\left(x_{1}, x_{2}, \cdots, x_{n}\right)$. Let $E_{A}$ denote the set of all functions of the form $r\left(x_{1}, x_{2}, \cdots, x_{n}, g_{1}\left(x_{1}, \cdots, x_{n}\right), \cdots, g_{m}\left(x_{1}, \cdots, x_{n}\right)\right)$ where $r$ is a rational function with complex coefficients whose denominator does not vanish identically when the substitution is made. We note that each element of $E_{A}$ defines a function algebraic over the field $\mathbf{F}_{\mathbf{0}}=\mathbf{C}\left(x_{1}, \cdots, x_{n}\right)$ of rational functions in $n$ variables with coeffcients in the complex numbers $\mathbf{C}$.

A solvable algebraic expression is any element of $E_{A}$ for any $\alpha$-program $A$. Let $\rho=$ $\left(f_{1}, f_{2}, \cdots, f_{s}\right)$ be a finite set of solvable algebraic expressions. We say that $\rho$ is computed by $A$, if each $f_{i} \in E_{A}$. Let $N_{\alpha}(\rho)$ be the minimum cost of any $\alpha$-program computing $\rho$. Clearly, $N_{\alpha}(\rho)$ is finite. For any such $\rho$, we can form the field $\mathbf{F}_{\mathbf{0}}(\rho)$ which is the algebraic extension of $\mathbf{F}_{\mathbf{0}}$ formed by adjoining the functions corredponding to the elements $f_{1}, \cdots, f_{s}$ of $\rho$.

Following [1], $\rho$ is said to be normal, if $\mathbf{F}_{\mathbf{0}}(\rho)$ is a finite Galois extension of $\mathbf{F}_{\mathbf{0}}$. In other words, $\rho$ is normal if $\rho$ generates the splitting field of some polynomial over $\mathbf{F}_{\mathbf{0}}$.

For any solvable group $G$, a cyclic Jordan-Hölder tower is a normal tower of groups

$$
G=G_{0} \triangleright G_{1} \triangleright \cdots \triangleright G_{m-1} \triangleright G_{m}=1,
$$

where $G_{i-1} / G_{i}$ is cyclic for each $1 \leq i \leq m$. Let $\nu(G)$ be the length $m$ of the shortest cyclic Jordan-Hölder tower for $G$.

The next result is from Ja'Ja' [1] which we state as a lemma: 
Lemma 1 [1] If $\rho$ is normal, then $N_{\alpha}(\rho)=\nu(G)$, where $G$ is the Galois group for $\mathbf{F}_{\mathbf{0}}(\rho)$ over $\mathbf{F}_{\mathbf{0}}$.

A $\beta$-program $B$ is a sequence of instructions of the form $z_{1} \leftarrow I_{1}, z_{2} \leftarrow I_{2}, \cdots, z_{m} \leftarrow I_{m}$, where $I_{i}$ are of the form $a_{i}^{1 / d_{i}}, \exp \left(a_{i}\right)$, or $\log \left(a_{i}\right)$, where $a_{i}=r_{i}\left(x_{1}, x_{2}, \cdots, x_{n}, z_{1}, \cdots, z_{i-1}\right)$ with $r_{i}$ is a rational function in $x_{1}, \cdots, x_{n}, z_{1}, \cdots, z_{i-1}$ with complex coefficients and $d_{i} \in$ $\mathbf{Z}^{+}$. We shall again always assume that the $r_{i}$ have been chosen so that the denominators of these functions do not vanish identically. Let $\tau(B)$ be the number of instructions which either take roots or exponentials. Let $g_{i}\left(x_{1}, x_{2}, \cdots, x_{n}\right)$ be the functions associated with variables $z_{i}$, defined exactly as in the case for $\alpha$-programs. Let $E_{B}$ denote the set of all functions of the form $r\left(x_{1}, x_{2}, \cdots, x_{n}, g_{1}\left(x_{1}, \cdots, x_{n}\right), \cdots, g_{m}\left(x_{1}, \cdots, x_{n}\right)\right)$ where $r$ is a rational function with complex coefficients whose denomincators do not vanish identically when the substituion is made.

Let $\rho=\left(f_{1}, f_{2}, \cdots, f_{s}\right)$ be a finite set of solvable algebraic expressions. We say that $\rho$ is computed by $B$, if each element $f_{i}$ of $\rho$ equals a function in $E_{B}$. Let $N_{\beta}(\rho)$ be the minimum $\tau(B)$ of any $\beta$-program $B$ computing $\rho$.

Our main result is the following theorem:

Theorem 1 If $\rho$ is normal, then $N_{\beta}(\rho)=\nu(G)$, where $G$ is the Galois group for $\mathbf{F}_{\mathbf{0}}(\rho)$ over $\mathbf{F}_{\mathbf{0}}$.

Corollary 1 If $\rho$ is normal, then $N_{\beta}(\rho)=N_{\alpha}(\rho)$.

Corollary 2 Let $\rho=\{f\}$, where $f=\sum_{1 \leq i \leq n} \sqrt{x_{i}}$. Then $N_{\beta}(\rho)=n$.

Remark It is an interesting open question whether $N_{\beta}(\rho)$ is equal to $N_{\alpha}(\rho)$ when $\rho$ is not required to be normal.

\section{Proof of Theorem 1}

Before proving the theorem, we introduce some terms in Differential Algebra (see [2], [3], [4]). A differential field is a field $k$ together with a set $\Delta=\left\{\delta_{i}\right\}$ of mappings $\delta_{i}$ : $k \rightarrow k$, called derivations, such that each $\delta_{i}$ satisfies the conditions $\delta_{i}(a+b)=\delta_{i}(a)+\delta_{i}(b)$, $\delta_{i}(a b)=\delta_{i}(a) b+a \delta_{i}(b)$, and $\delta_{i}\left(\delta_{j}(a)\right)=\delta_{j}\left(\delta_{i}(a)\right)$ for all $\delta_{i}, \delta_{j} \in \Delta, a, b \in k$. For example, $\mathbf{F}_{\mathbf{0}}$ can be considered a differential field when we use the derivations $\Delta=\left\{\delta_{1}, \cdots, \delta_{n}\right\}$ where $\delta_{i}(f)=\partial f / \partial x_{i}$. In this paper we are concerned only with differential fields that come from fields of differentiable functions $a$ and that are extensions of this differential field. These extensions will be gotten by adjoining elements that can be interpreted as functions on some suitable region in complex $n$-space $\mathbf{C}^{n}$. We will use $\mathbf{K}_{\mathbf{0}}$ to denote the 
differential field obtained from the field $\mathbf{F}_{\mathbf{0}}$ equipped with these standard derivations $\Delta$. Note that if $\mathbf{K}$ is a differential field containing $\mathbf{K}_{\mathbf{0}}$ and if $a \in \mathbf{K}$, then the field obtained by adjoining $\exp (a)$ to $\mathbf{K}$ gives a differential field. The element $\exp (a)$ will satisfy the differential equations $\delta_{i}(\exp (a))=\delta_{i}(a) \cdot \exp (a)$ for $i=1, \cdots, n$. Similarly, the adjoining of $\log (a)$ gives a differential field and the element $\log (a)$ satisfies $\delta_{i}(\log (a))=\delta_{i}(a) / a$ for $i=1, \cdots, n$. We also note that if $\rho=\left(f_{1}, \cdots, f_{s}\right)$ is a set of solvable algebraic expressions (or, more generally, any set of algebraic functions), the derivations $\Delta$ can be extended uniquely to derivations on $\mathbf{F}_{\mathbf{0}}(\rho)$ ([4], Lemma 1, p.90).

The classical Galois theory for field theory can be extended to a differential Galois theory for differential fields (See [3] and [4] for definitions and discussions of these concepts; [2] contains an excellent exposition of the theory in the case of only one derivation and the essential results extend, mutatis mutandi to the case of several derivations). This galois theory can be used to study the structure of the solutions of a system of partial linear differential equations, provided that the equations generate a differential ideal of finite linear dimension or, equivalently (see [4], Chapter IV.5), the solution space is a finite dimensional vector space (i.e., the system is holonomic). This is the case for the equations defining exponentials and logarithms (see [3] and [4]). To avoid possible confusions, we will reserve the term Galois group for the classical Galois group, and use the term differential Galois group when differential fields are being discussed. It should be noted, though, that if $k_{1}$ is an algebraic extension of $k_{0}$, a differential field of characteristic zero, then since all derivations on $k_{0}$ can be extended uniquely to derivations on $k_{1}$, we can identify the Galois group of $k_{1}$ over $k_{0}$ with the differential galois group of $k_{1}$ over $k_{0}$ (with respect to these derivations). To see this note that any differential automorphism is by definition a usual automorphism. Conversely, for any automorphism $\sigma$ of $k_{1}$ over $k_{0}$ and any derivation $\delta$ of $k_{1}$ that leaves $k_{0}$ invariant, we have that $\sigma^{-1} \circ \delta \circ \sigma$ is a derivation of $k_{1}$ agreeing with $\delta$ on $k_{0}$. Uniqueness implies that they must be equal on all of $k_{1}$ and so $\sigma$ must be a differential automorphism. This remark allows us to apply results concerning differential galois theory to the galois theory of algebraic extensions of differential fields.

To prove Theorem 1, we first show that if $\mathbf{F}_{\mathbf{0}}(\rho)$ is contained in a certain tower of differential fields, then there is a tower of algebraic extension fields of no greater length containing $\mathbf{F}_{\mathbf{0}}(\rho)$. This result (Lemma 2 below) is at the heart of the proof for Theorem 1.

Let $\mathbf{K}_{\mathbf{0}} \subset \mathbf{K}_{1} \subset \mathbf{K}_{\mathbf{2}} \subset \ldots \subset \mathbf{K}_{\mathrm{m}}$ be a tower of differential fields, where each $\mathbf{K}_{\mathbf{i}}$ is obtained from $\mathbf{K}_{\mathbf{i}-1}$ by adjoining an element $u_{i} ; u_{i}$ is either $\exp \left(a_{i}\right)$ or $\log \left(a_{i}\right)$ with $a_{i} \in \mathbf{K}_{\mathbf{i}-1}$. Let $I$ be the set of $1 \leq i \leq m$ such that $u_{i}$ is $\exp \left(a_{i}\right)$. We recall from differential Galois theory that in this case each $\mathbf{K}_{\mathbf{i}}$ is a Picard-Vessiot extension of $\mathbf{K}_{\mathbf{i}-1}$. Furthermore, it is known (see [3, Section 4], or [4, Chapter VI.6]; [2, Lemmas 3.9 and 
3.10] contains simillar results for the case of one derivation) that, if $i \in I$, the differential Galois group of $\mathbf{K}_{\mathbf{i}}$ over $\mathbf{K}_{\mathbf{i}-1}$ is an algebraic subgroup of $\mathbf{C}^{*}$, the multiplicative group of non-zero complex numbers, and if $i \notin I$, then the differential Galois group of $\mathbf{K}_{\mathbf{i}}$ over $\mathbf{K}_{\mathbf{i}-1}$ is an algebraic subgroup of $\mathbf{C}^{+}$, the additive group of complex numbers. Finally, we note that the proper algebraic subgroups of $\mathbf{C}^{*}$ are precisely the finite cyclic groups and the only proper algebraic subgroup of $\mathbf{C}^{+}$is the trivial group. This can be seen by noting that a proper Zariski closed subset of either of these two groups must be finite and that in the first case, we will have a finite multiplicative subgroup of a field and in the second case we will have a finite subgroup of a torsion free group.

Lemma 2 If $\mathbf{F}_{0}(\rho) \subset \mathbf{K}_{\mathbf{m}}$ then $\nu(G) \leq|I|$.

Proof Let $\mathbf{F}_{\mathbf{i}}=\mathbf{F}_{0}(\rho) \cap \mathbf{K}_{\mathbf{i}}$ for $1 \leq i \leq m$. Then $\mathbf{F}_{\mathbf{m}}=\mathbf{F}_{0}(\rho)$. Note that $\mathbf{F}_{\mathbf{0}}=\mathbf{F}_{0}(\rho) \cap \mathbf{K}_{\mathbf{0}}$. Let $H_{i}$ be the differential Galois group of $\mathbf{K}_{\mathbf{i}}$ over $\mathbf{K}_{\mathbf{i}-1}$. We claim that the following statement is true for $1 \leq i \leq m$ :

Fact $1 \mathbf{F}_{\mathbf{i}}$ is a Galois extension of $\mathbf{F}_{\mathbf{i}-1}$.

To prove this fact, let $\mathbf{E}_{\mathbf{i}}$ be the subfield of elements of $\mathbf{K}_{\mathbf{i}}$ algebraic over $\mathbf{K}_{\mathbf{i}-1} \cdot \mathbf{E}_{\mathbf{i}}$ is a differential field and is left invariant by all elements of $H_{i}$. Therefore the differential Galois group of $\mathbf{K}_{\mathbf{i}}$ over $\mathbf{E}_{\mathbf{i}}$ is a normal subgroup of $H_{i}$ and so $\mathbf{E}_{\mathbf{i}}$ is a Galois extension of $\mathbf{K}_{\mathbf{i}-1}$. Note that $\mathbf{F}_{\mathbf{i}}=\mathbf{E}_{\mathbf{i}} \cap \mathbf{F}_{0}(\rho)$. Let $p(x)$ be a polynomial with coeffcients in $\mathbf{F}_{\mathbf{i}-1}$. If $p(x)=0$ has a root in $\mathbf{F}_{\mathbf{i}}$, it must split in both $\mathbf{E}_{\mathbf{i}}$ and $\mathbf{F}_{0}(\rho)$ (since $\mathbf{F}_{0}(\rho)$ is a fortiori normal over $\left.\mathbf{F}_{\mathbf{i}-1}\right)$. Therefore $p(x)=0$ splits in $\mathbf{F}_{\mathbf{i}}$ and so $\mathbf{F}_{\mathbf{i}}$ is a Galois extension of $\mathbf{F}_{\mathbf{i}-1}$.

Now let $J_{i}$ be the Galois group of $\mathbf{F}_{\mathbf{i}}$ over $\mathbf{F}_{\mathbf{i}-1}$. We claim that the following statement is true:

Fact 2 For $1 \leq i \leq m, J_{i}$ is the trivial group if $i \notin I$, and a cyclic group if $i \in I$.

To prove this fact, consider the field $\mathbf{K}_{\mathbf{i}-1} \cdot \mathbf{F}_{\mathbf{i}}$. This is a subfield of $\mathbf{K}_{\mathbf{i}}$. Since $H_{i}$ is an abelian group, all of its subgroups are normal, so $\mathbf{K}_{\mathbf{i}-1} \cdot \mathbf{F}_{\mathbf{i}}$ is a normal extension of $\mathbf{K}_{\mathbf{i}-1}$ whose differential Galois group $L_{i}$ is the quotient of $H_{i}$ by a closed subgroup of $H_{i}$. Furthermore, since $\mathbf{K}_{\mathbf{i}-1} \cdot \mathbf{F}_{\mathbf{i}}$ is a finite extension of $\mathbf{K}_{\mathbf{i}-1}, L_{i}$ is finite and thus coincides 
with the Galois group of this extension. If $i \notin I$, then $H_{i}$ is either $\mathbf{C}^{+}$or the trivial group. The only finite quotient of either of these groups by a closed subgroup is trivial. If $i \in I$, then $H_{i}$ is either $\mathbf{C}^{*}$ or a finite cyclic group. The only possible finite quotients of these groups by closed subgroups are cyclic. To finish the proof of Fact 2, we note that $\mathbf{K}_{\mathbf{i}-1} \cap \mathbf{F}_{\mathbf{i}}=\mathbf{F}_{\mathbf{i}-1}$ and so the Galois group $J_{i}$ of $\mathbf{F}_{\mathbf{i}}$ over $\mathbf{F}_{\mathbf{i}-1}$ is isomorphic to $L_{i}$ (see [5, Corollary, p. 400] or [4, Chapter VII, Theorem 1.12]; [2, Lemma 5.10] is a related result but deals only with the case of one derivation.)

We can now finish the proof of Lemma 2. Let $G_{i}$ denote the group of automorphisms of $\mathbf{F}_{0}(\rho)$ leaving $\mathbf{F}_{\mathbf{i}}$ fixed. By Facts 1 and 2, one concludes from the Galois theory that the series $G=G_{0}, G_{1}, G_{2}, \ldots, G_{m}=1$ forms a cyclic Jordan-Hölder tower, with $G_{i-1} / G_{i}$ being isomorphic to $J_{i}$. Deleting all $i \notin I$, we have a tower of length $|I|$. Hence $\nu(G) \leq|I|$.

We now turn to the proof of Theorem 1 . Observe that $N_{\beta}(\rho) \leq N_{\alpha}(\rho)$, which is no greater than $\nu(G)$ by Lemma 1 . Thus, we only need to prove that $N_{\beta}(\rho) \geq \nu(G)$.

Let $B$ be any $\beta$-program for computing $\rho$. Without loss of generality, we may assume that no root-taking operations are used in $B$, as we can replace any instruction $z-r^{1 / d}$ by two instructions $y \leftarrow(\log r) / d, z \leftarrow \exp (y)$ without changing the value of $\tau(B)$. Let

the instructions be $z_{1} \leftarrow I_{1}, z_{2}-I_{2}, \cdots, z_{m} \leftarrow I_{m}$. Let $g_{i}\left(x_{1}, x_{2}, \cdots, x_{n}\right)$ be the functions associated with variables $z_{i}$.

For $1 \leq i \leq m$, let $\mathbf{K}_{\mathbf{i}}$ be the differential field obatained by adjoining $g_{i}$ to $\mathbf{K}_{\mathbf{i}-1}$. By definition, the functions of $E_{B}$ correspond to elements of $\mathbf{K}_{\mathbf{m}}$ and $\mathbf{F}_{\mathbf{0}}(\rho) \subset \mathbf{K}_{\mathbf{m}}$. By Lemma 2, this implies $\nu(G) \leq \tau(B)$. This proves $\nu(G) \leq N_{\beta}(\rho)$, and completes the proof of Theorem 1. Corollary 1 follows immediately from the theorem and Lemma 1.

To prove Corollary 2, we note that $\rho$ is normal and the Galois group $G$ of $\mathbf{F}_{\mathbf{0}}(\rho)$ over $\mathbf{F}_{\mathbf{0}}$ is isomorphic to $\mathbf{Z}_{\mathbf{2}}{ }^{n}$. ¿From the result in [1, 7] (see [7, p. 399, Lemma 3.2]), $\nu(G)$ is equal to the number of torsion orders of $G$ which is cleary $n$. Corollary 2 follows from the theorem immediately.

\section{References}

[1] J. Ja' Ja', "Computation of algebraic functions with root extractions," Proceedings of 22nd IEEE Symposium on Foundations of Computer Science, 1981, 95-100.

[2] I. Kaplansky, An Introduction to Differential Algebra, Hermann, Paris, 1957. 
[3] E. R. Kolchin, "Picard-Vessiot theory of partial differential fields," Proceedings of the American Mathematical Society, 3 (1952), 596-603.

[4] E. R. Kolchin, Differential Algebra and Algebraic Groups, Academic Press, New York, 1973.

[5] S. Lang, Algebra, Addison-Wesley, Menlo Park, California, 1984 (Second Edition).

[6] N. Pippenger, "Computational complexity of algebraic functions," Journal of Computer and System Sciences 22 (1981), 454-470.

[7] N. Pippenger, "Corrections to 'Computational complexity of algebraic functions'," Journal of Computer and System Sciences 37 (1988), 395-399.

[8] M. Shamos and G. Yuval, "Lower bounds from complex function theory," Proceedings of 17th IEEE Symposium on Foundations of Computer Science, 1976, 268-273. 